\title{
Performance Visualizations using XML Representations
}

\author{
Yijun $\mathrm{Yu}^{*}$ Kristof Beyls ${ }^{\dagger}$ Erik H. D'Hollander ${ }^{\dagger}$ \\ ${ }^{*}$ CS, University of Toronto. 40 St George Street, M5S2E4 Toronto, Canada \\ ${ }^{\dagger}$ ELIS, University of Ghent. 41, 9000 Ghent, Belgium
}

\begin{abstract}
The intermediate representation (IR) forms the information exchanged among different passes of program compilation. The intermediate format proposed for extensibility and persistence is written in XML. In this way, the program transformations that were internal to the compiler become visible. The hierarchical structure of XML makes a natural representation for the abstract syntax tree (AST). A compiler can parse the program source into an IR, then output it as an XML document. Separated by orthogonal namespaces, other IRs are also presented in the same XML document, gathering program information such as dependence vectors, transforming matrices, iteration spaces dependence graphs and cache reuse distances. This XML document can be exchanged between the compiler and program visualizers for parallelism and locality. Keywords-
\end{abstract}

intermediate representations, XML, performance visualizations

\section{Introduction}

Designers of compiler systems have employed the intermediate representation (IR) to systematically define the data interface between its different subsystems $[1,6,4]$. An IR includes the most common data structures used in the compiler. The high level data structures include the abstract syntax tree, the symbol and the type table, the call graph and the dependence graph, and the low level data structures include the task graph and the instruction tuples.

In order to exchange information between different passes and to support flexible combination of compile options, the IR is made persistent through an intermediate format like SUIF in the SUIF compiler [15], and Lcode in the IMPACT compiler [7]. Similar efforts are also made to serialization of Java data structure through the java.io.Serializable interface [10].

Earlier efforts like ASDL [14] has been made to pickle or marshal the tree-like IR defined within individual compiler like SUIF. After writing several hundred lines of ASDL definition, thousands line of code implementing the API for pickling are generated. The API supports the sharing of information among different compilers or between a compiler and another programming tool.
Experience with ASDL in 1CC [8] has shown how to retrofit an existing compiler by separating its $\mathrm{C}$ parser and the multi-platform code generator into two components communicating only through ASDL. Afterwards new optimizer components could be inserted without changing the two parts.

Both the designers and the users of ASDL have notified that the drawback of using ASDL is a duplication of data structure in memory $[14,8]$. To improve the efficiency, the Aterm [13] targeting at minimize the storage of trees in more efficient binary formats.

With the advent of XML [3] as an information exchange standard, almost every corner of the software industry is inspired to share the once-incompatible information to the other XML enabled applications. This trends already deeply touched the compiler industry. Any XML document, in the first place, has to be valid against its DTD(document type definition) by an XML parser. Another example is the GCCXML Kitware project, where XML is adopted to output procedural information [9]. Both the Java serialization and the ASDL pickle can further be converted from and to the XML format [8].

However small, both ASDL and Aterm still have to occupy memory as additional overhead when retrofit an existing compiler, while pure event-based XML parser SAXparser does not. In this paper, we propose a thorough use of XML in the compiler infrastructure. That is, to exchange all the IR data structures in XML as needed. This will support persistence of the compiler internal information and make it extensible for outside tools to share the information with the compiler. As will be shown in the paper, not only tree-like AST can be represented, various IR data structures including complex dependence graphs can be expressed in XML. The use of orthogonal namespaces for various data structures makes it possible to bind related information in a single XML document. The apparent huge requirement for XML storage can be alleviated by a stand-alone compression tool.

The remainder of the paper is given as follows: section 2 discusses the reason to XMLize the common IR used in most of the compilers; section 3 overviews the compiler system combined with external transforming tools and vi- 
Table 1: The name-spaces for our compiler IR

\begin{tabular}{ll} 
namespace & representation \\
\hline ast & an abstract syntax tree(AST) \\
yaxx & a YACC parsing tree \\
par & an identified parallel or sequential loop \\
isdg & an iteration space dependence graph \\
hotspot & performance bottleneck locations \\
trace & an execution trace of memory instructions \\
cache & parameters for simulating a cache \\
rdv & a reuse distance vector
\end{tabular}

sualizers; section 4 visualize other program information like loop dependence and cache behavior by sharing information between the compiler and various visualizers; section 5 concludes the work with future perspectives.

\section{Representations in XML}

An XML document for the intermediate representation (IR) is a tree of XML tags. In our design, an IR document has the following form:

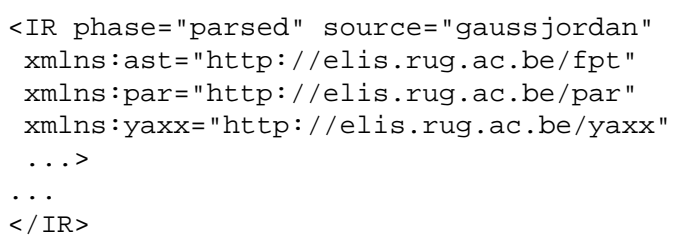

The root "IR" tag of the document tree has two basic attributes: "phase" indicates which compiling or visualizing phase the IR belongs to; "source" indicates the name of the source program under investigation. As the document root, it carries a number of uniform resource identifiers for orthogonal name-spaces, e.g. see table 1. Though they are not a complete set of IR used in program transformations and visualizations, the extensibility of XML opens possibility for name-spaces of additional applications.

Each child XML tag is prefixed with the name-space corresponding to a certain intermediate representation. For example, the abstract syntax tree has an ast: prefix, the parsing tree has a yaxx: prefix, the parallelizable loop has a par: prefix, etc.

Global data structures are put as the immediate subtrees of the document root. E.g. ast:N_PROGRAM as the root of an AST; the name of the starting rule yaxx:executable_program as the root of a parsing tree, a trace: sequence as the root of a program execution trace, etc.

Local data structures are put as the children of an AST node corresponding to its scope. For example, the dependence test annotates each parallel DO loop "ast:S_DO" with the parallel information:

<ast:S_DO><par:true/> $\ldots</$ ast:S_DO>
Similarly, tag isdg: graph is a child of the outermost loop of a loop nest <ast:S_DO>, denoting an iteration space dependence graph. Other information, such as hot spot: histogram which contains statistic information for cache miss patterns can be attached to any statement in the AST, either a program unit, a loop nest or even a single memory instruction. In this way, program semantic information are othogonally associated with the AST in one XML document.

\section{Extending the system architecture}

Using XML to represent the IR in a compiler, we aim to extend the compiler with the ability to exchange information with external tools, such as Omega calculator [11] and program visualizers including ISV [17] and CacheVis [16]. The design of the integrated system is shown in figure 1 . As a source-to-source optimizing compiler, FPT is sepa-

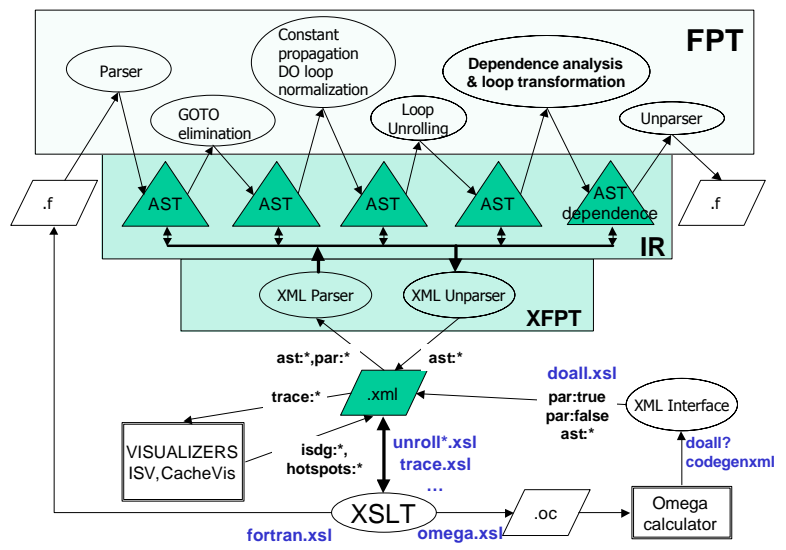

Figure 1: The extended system architecture.

rated into several phases: the first phase parse a source Fortran program into AST; then each phase either changes the AST or creates additional IR; the last phase unparse the AST back to a target Fortran program.

The separation of phases was internal to FPT before introducing the XML interface: XML parser and XML unparser. They bridge between the IR and the XML document, which is then given to external tools, namely, XSLT, Omega and Visualizers.

The XSLT is a collection of XML transformers: unroll*.Xsl performs loop unrolling transformation which has been implemented in FPT. Now it is performed purely in XSLT, showing the potential to implement a compiler transformation outside the compiler; fortran.xs 1 just produces the Fortran source code from the syntax information in the IR document, which is similar to FPT unparser. But it does not require the document to be loaded into FPT and does provide a convenient debugging tool for 
checking the correctness of transformations; omega . xs I extracts the iteration space and dependence equations from an AST as input to the Omega calculator, and doal1 . xs 1 interfaces with Omega calculator to annotate the parallel loop loops in the AST; finally, trace.xsl creates an instrumented program that allows dependence analyzers in ISV and cache simulator in CacheVis to visualize the dependence graph and cache miss hot spots.

\section{Program Visualizations}

The AST in XML form can be obtained from the XMLenabled parser such as YAXX, then visualized either by using commercial XML editors such as XMLSpy or opensource graph layout tools such as GraphViz [5] and Vcg [12]. Besides the AST, other semantics information can also be visualized.

For performance optimization, two kinds of program characteristics are considered extremely important. One is the parallelism, the other data locality. The semantics information for studying parallelism is data dependence, while the information for studying the cache behavior is reuse distances [2] in cache stack history. The data dependences can be visualized using a loop dependence visualizer ISV [17]; the data locality can be visualized using a cache visualizing tool CacheVis [16]. The integration glue for them with the compiler is naturally XML.

\subsection{AST in XML for visualization}

Abstract syntax tree(AST) is the most commonly used IR for the program syntax. The syntax of high-level programming languages are expressed in production rules in Backus Naur Form (BNF). On reduction of a production rule, the right-hand sided terms become the children of the left-hand sided term in a syntax tree.

We adapted bison, an open-source variant of YACC, to produce a DTD from the YACC grammar and a parsing tree in the DTD-compliant XML corresponding to a program follows the grammar. The "start" non-terminal becomes the root of the XML document. A terminal node is always a leaf node in the XML tree. A non-terminal with non-empty production rule have the RHS nodes as its children.

A parsing tree might be used as an AST before several concerns are addressed.

- The parsing tree from YACC grammar are usually too deep because a repetitive structure like a list is often represented by recursive production rules:

$$
\text { List : Item | Item List }
$$

However, we can make it more readable by simplifying the production rule using a feature of document type definition(DTD) element:

\section{$<$ ! ELEMENT List $(($ Item $)+)>$}

- Secondly it is redundant to have two rules like "A:==B, B:==C" while the first rule is the only rule defining A. It can be removed by replacing every occurrence of $\mathrm{A}$ into $\mathrm{B}$.

After the above simplifications, a grammar does not lose its expressiveness. To express a Fortran program, its AST is expressed by the following document type definition:

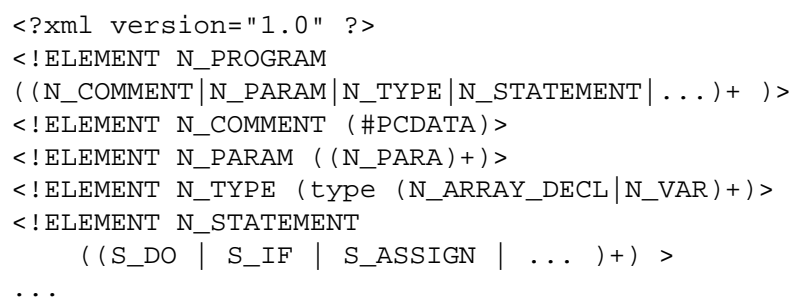

The XML tags correspond to different types of AST node. Consider a small Fortran program as follows.

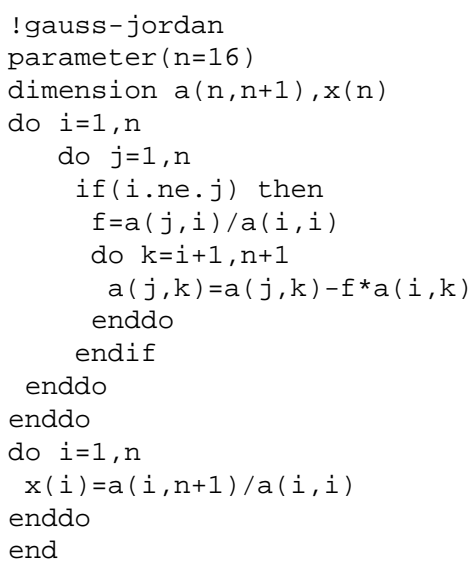

Its AST is generated as an XML document shown in XMLSpy ( Figure 2a). The AST can also be transformed by XSLT into GraphViz [5] visualizer as an up-side-down tree (Figure 2b).

\subsection{Loop dependence visualization}

As long as the iteration space dependence graph is generated, one can visualize it in the ISV [17]. The tool shows the maximum parallelism in the data-flow execution of the loop and finds a suitable transformation interactively. First a nested loop is instrumented with output statement by trace.xsl to generate the symbolic memory trace. When executing the instrumented program, a memory instruction trace is generated. For instance, the instrumented Gauss-Jordan outputs the following trace:

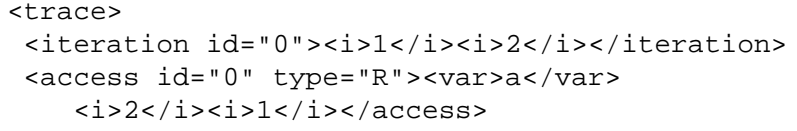




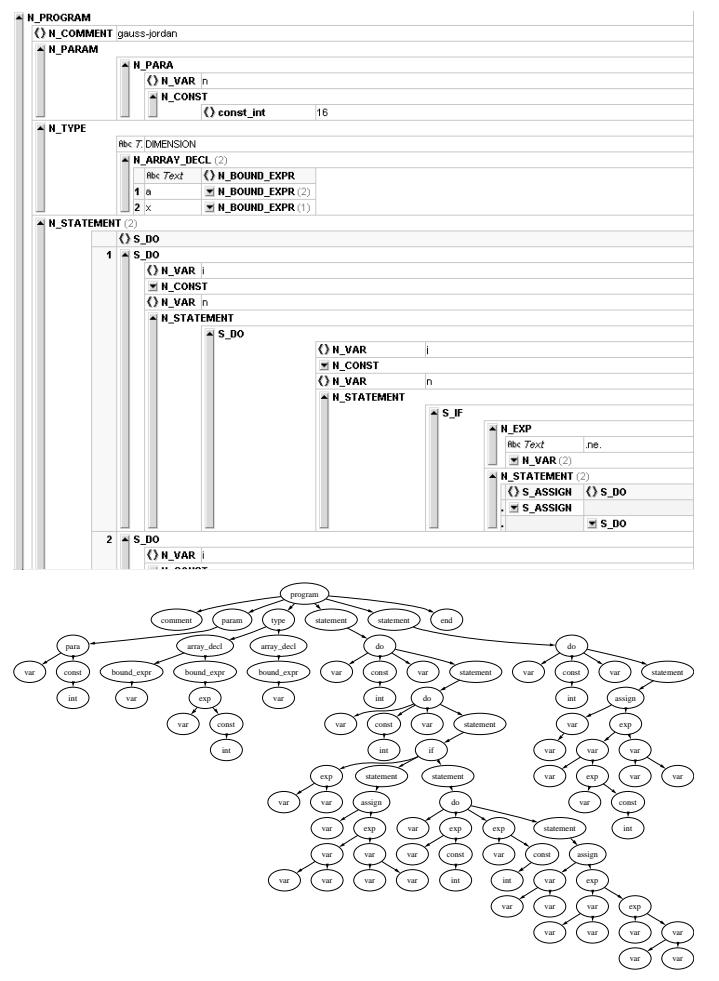

Figure 2: Visualizing the AST.
... (83 dependence edges)

$</$ ISDG $>$

The ISDG is displayed in the three dimensional iteration space in ISV, as shown in Figure 3. From the graph, one sees no dependences in each $i$ plane, i.e. the J, K loops are parallelizable.

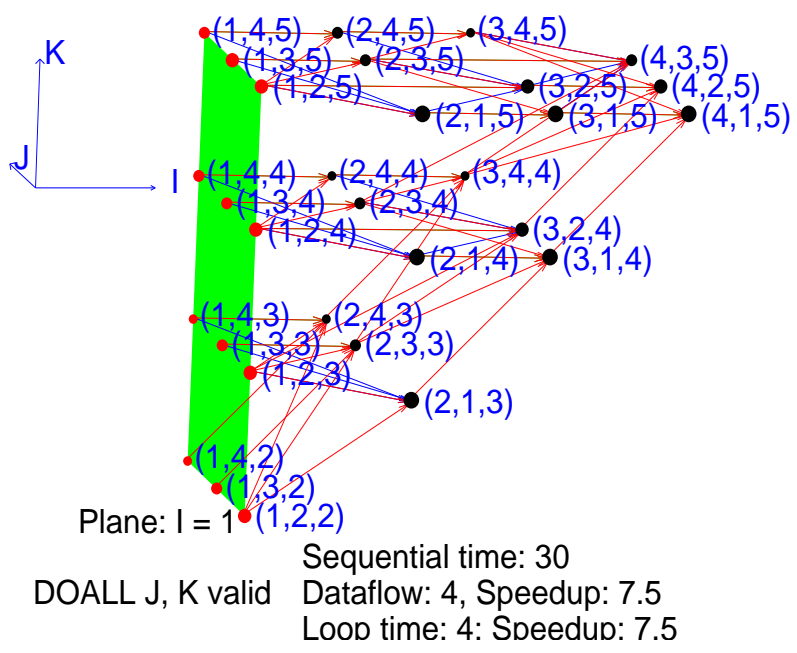

Figure 3: Gauss-Jordan visualized by the ISV in [17].

\subsection{Cache miss visualization}

Similar to the trace instrumentation done in the ISV, the memory trace is used to simulate a cache to generate the information for visualizing the distribution of cache misses of an program execution [16]. In "memory trace.dtd", instead of showing the variable and their subscripts of each access, its actual memory address is output.

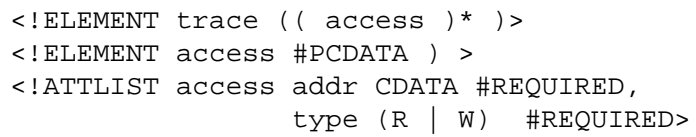

Here only iterations with non-empty accesses are output. An access without index is a scalar, e.g., $f$.

Then the iteration space visualizer analyzes the dependences using the trace and creates the following iteration space dependence graph:

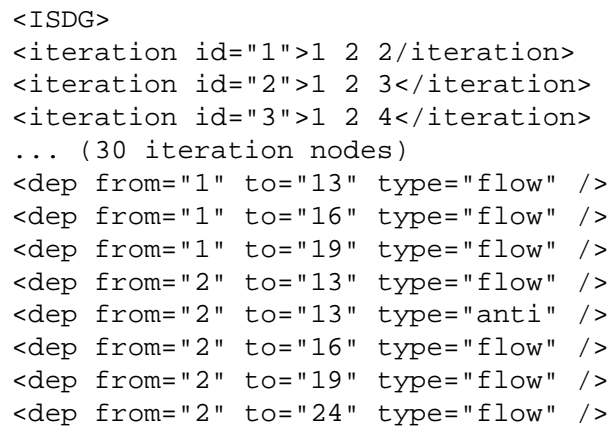

Besides, the cache parameters are given to the cache simulator as an configuration file. E.g. a $1 \mathrm{~KB}$ directmapped cache with cache line size 32 bytes is given.

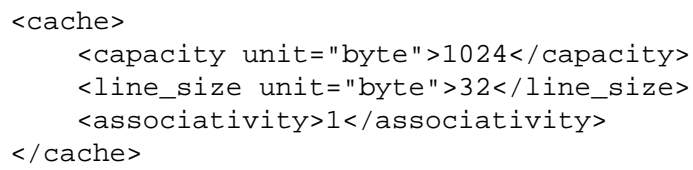

The visualizer computes the histogram from the cache miss trace in the following format.

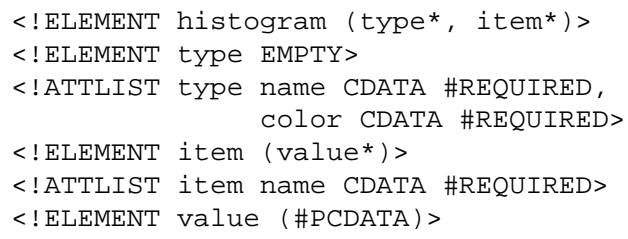




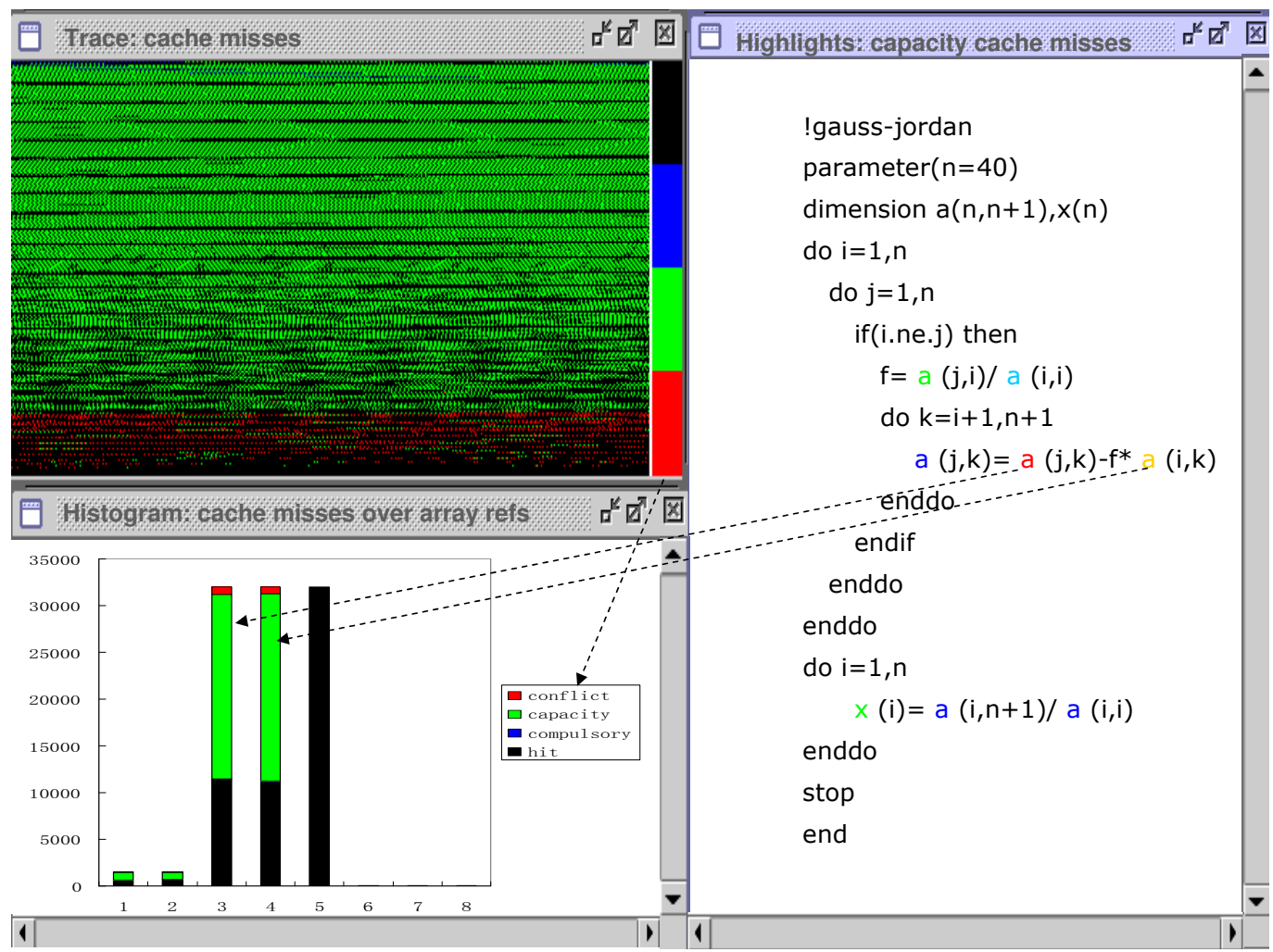

Figure 4: Gauss-Jordan in the cache visualizer [16]. Upper-left window shows a trace of cache misses wrapped at the horizontally border; lower-left window shows a histogram chart of cache misses over array references, its legend also explains the colors used (see upper), its X-axis are array references numbered by the lexicographical order in code (see right); the array references in the right window are spectrumly colored (blue to red stands for small to large) by the intensity of capacity misses.

Both traces and histograms of the Gauss-Jordan program are displayed in figure 4 . The graphs show the dominant cache misses are capacity miss, and most capacity misses occur to the 3 rd and 4 th references in the innermost loop.

\subsection{Cache reuse distance visualization}

Besides the traces and histograms, the cache visualizer also shows the distribution of the reuse distances [2] among the hot spots in the source code. When consecutive reuses of data occur far appart in time, there is a high probability for a cache miss.

First the hot spots are defined by the following DTD.

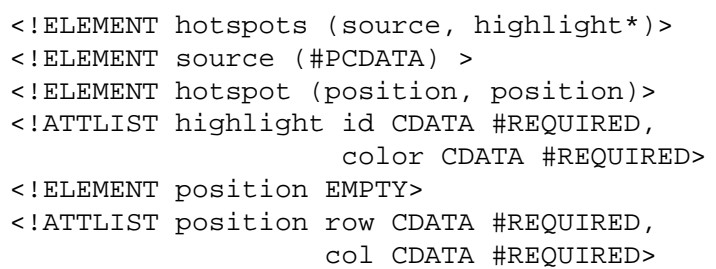

A hot spots document indicates the source file name by source child. Each hot spot is a region from $\left(\operatorname{row}_{1}, \mathrm{col}_{1}\right)$ to $\left(\mathrm{row}_{2}, \mathrm{Col}_{2}\right)$ in the source code. Thus the source code is visualized with the hot spots highlighted in specified colors. The distribution of reuse distance in the source code is visualized by colors in the right window in figure 4 .

Another way of presenting the hot spots is using a graph layout tool vcg [12]. First the cache simulator outputs the document defined by the following DTD:

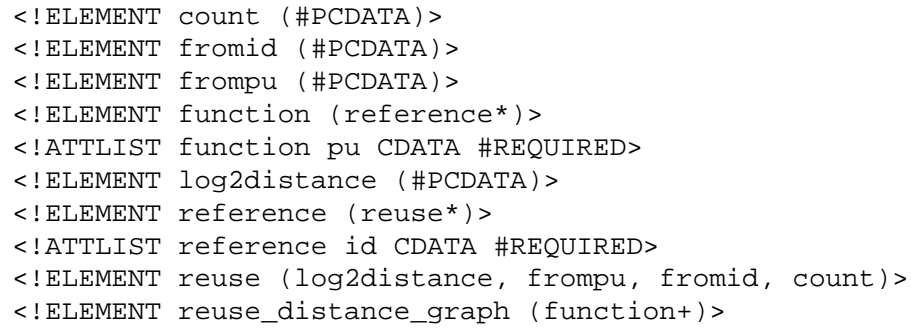

Each instruction has a histogram counting the memory accesses over different $\log 2$ reuse distances. The reused instructions with long reuse distances that generate capacity misses are filtered, then these instructions pairs are displayed as directed graph in vcg.

The long reuse distances of health, one of the Olden benchmark programs, are visualized in figure 5 . One can see these hot spots coincide with the performance observation comments in the program.

\section{Conclusion}

Compiler transformations use intermediate representations which were internal to the compiler. The intermediate representations such as abstract syntax tree, dependence distances, transforming matrices, loop dependence graphs, memory traces and cache reuse distances can now be exchanged in XML, because XML is extensible to ex- 


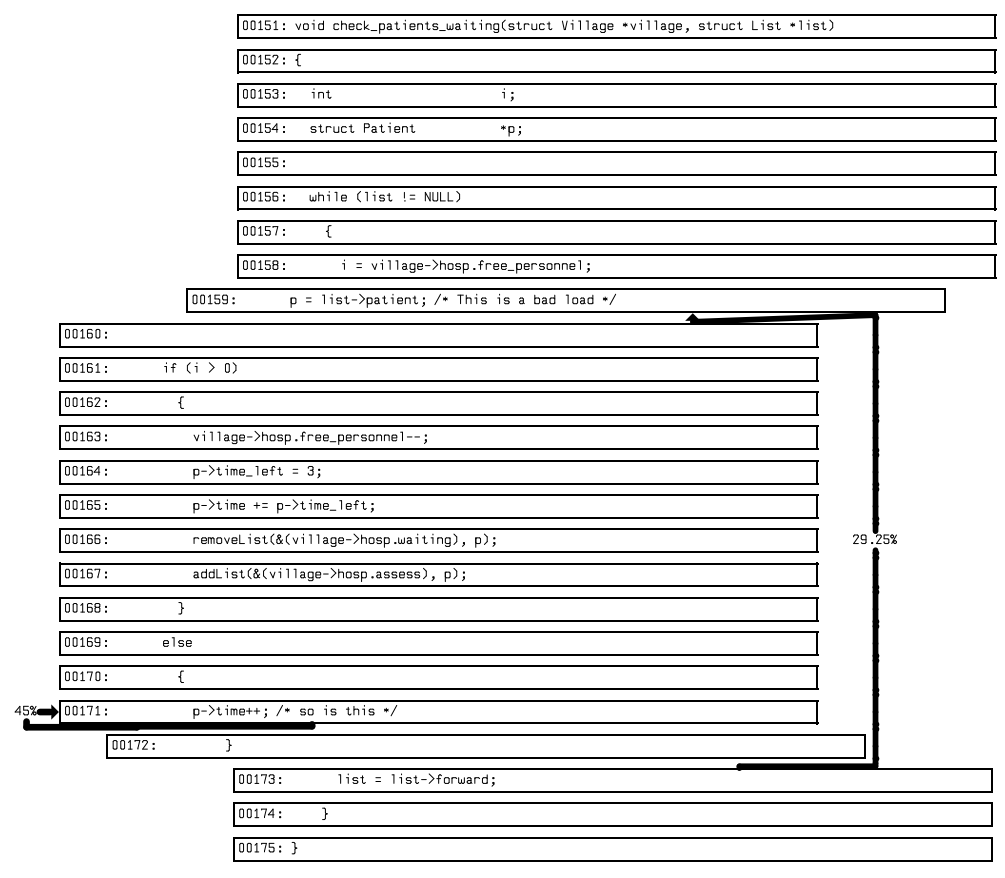

Figure 5: The long reuse distances visualized as hot spots

press any structural data in DTD or Schema. An IR of existing compiler can be re-engineered incrementally through adding orthogonal namespaces to support additional compiler transformations. Transformations can be done even outside the compiler to exchange information with various XML-enabled programming tools. Thus, XML becomes a glue to form an open research environment by seamlessly binding the compiler with external transformation tools such as Omega calculator and visualizers for loop dependences and cache reuse distances.

\section{References}

[1] A. V. Aho, R. Sethi, and J. D. Ullman. Compilers, Principles, Techniques, and Tools. Addison, 1986. ISBN 0-201-10088-6.

[2] K. Beyls and E. D‘Hollander. Reuse distance as a metric for cache behavior. In T. Gonzalez, editor, Proceedings of the IASTED International Conference on Parallel and Distributed Computing and Systems, pages 617-622, Anaheim, California, USA, 82001. IASTED.

[3] T. Bray, J. Paoli, and C. Sperberg-MacQueen. Extensible markup language. Technical report, http://www.w3.org/TR/REC-xml, feb 1998.

[4] K. A. Faigin, S. A. Weatherford, J. P. Hoeflinger, D. A. Padua, and P. M. Petersen. The Polaris internal representation. International Journal of Parallel Programming, 22(5):553-586, 1994.

[5] E. R. Gansner, E. Koutsofios, S. C. North, and K.-P. Vo. A technique for drawing directed graphs. IEEE TRANS. SOFTW. ENG., 19(3):214-230, May 1993.

[6] M. Girkar and C. D. Polychronopoulos. The hierarchical task graph as a universal intermediate representation. International Journal of Parallel Programming, 22(5):519-551, 1994.

[7] B. Goldberg, H. Kim, V. Kathail, and J. Gyllenhaal. The Trimaran compiler infrastructure for instruction level parallelism research.
Technical report, Hewlett-Packard Laboratories, University of Illinois, NYU, 1998.

[8] D. R. Hanson. Early experience with ASDL in lcc. Software Practice and Experience, 29(5):417-435, 1999.

[9] Kitware. The GCC-XML extension. Technical report, http://public.kitware.com/GCC_XML.

[10] S. Microsystems. Java object serialization specification, revision 0.9. Technical report, Sun Microsystems, 1996.

[11] W. Pugh. A practical algorithm for exact array dependence analysis. $C A C M, 35(8): 102-114$, AUG 1992.

[12] G. Sander. Graph layout through the VCG tool. Lecture Notes in Computer Science, 894:194-205, 1995.

[13] M. van den Brand, H. de Jong, P. Klint, and P. Olivier. Efficient annotated terms. Software, Practice and Experience, 30(3):259$291,2000$.

[14] D. C. Wang, A. W. Appel, J. L. Korn, and C. S. Serra. The zephyr abstract syntax description language. Technical Report TR-55497, http://www.zephyr.com, Oct. 1997.

[15] R. Wilson, R. French, C. Wilson, S. Amarasinghe, J. Anderson, S. Tjiang, S. Liao, C. Tseng, M. Hall, M. Lam, and J. Hennessy. SUIF: An infrastructure for research on parallelizing and optimizing compilers. ACM SIGPLAN Notices, 29(12), dec 1996.

[16] Y. Yu, K. Beyls, and E. D'Hollander. Visualizing the impact of the cache on program execution. In Proceedings of Fifth International Conference on Information Visualization, pages 336-341, London, England, July 2001.

[17] Y. Yu and E. D'Hollander. Loop parallelization using the 3D Iteration Space Visualizer. Journal of Visual Languages and Computing, 12(2):163-181, April 2001. 